Research Article

\title{
Occurrence of Biofilm Formation in Serratia fonticola and Pantoea sp. Isolates among Urinary Catheterized Patients
}

\author{
Shaimaa Obaid Hasson ${ }^{1}$, Adnan Hamad Al-Hamadani ${ }^{2}$, Ibtisam Habeeb Al-Azawi ${ }^{2}$ \\ ${ }^{1}$ Department of Microbiology, College of Veterinary, Al-Qassim Green University, Iraq. \\ ${ }^{2}$ Department of Medical Microbiology, College of Medicine, Al-Qadisiyah University, Iraq. \\ Corresponding author. E-mail: shaimaaobead@gmail.com
}

Received: Jun. 25, 2018; Accepted: Jul. 19, 2018; Published: Sep. 29, 2018.

Citation: Shaimaa Obaid Hasson, Adnan Hamad Al-Hamadani, and Ibtisam Habeeb Al-Azawi, Occurrence of Biofilm Formation in Serratia fonticola and Pantoea sp. Isolates among Urinary Catheterized Patients. Nano Biomed. Eng., 2018, I0(3): 295-304.

DOI: 10.5101/nbe.v10i3.p295-304.

\begin{abstract}
Serratia fonticola and Pantoea sp. are gram negative bacteria belonging Enterobactericeae, which were considered opportunistic pathogens and resulted in a great number of cases of nosocomial infections with serious problems of multi-drug resistance, leading to increasing morbidity and mortality rate. Recently, they were recorded as biofilm producers. There were only a few studies about the capability of these bacteria of forming biofilm. So our aim was evaluate the occurrence of Serratia fonticola and Pantoea sp. biofilm former phenotypically and genetically with the determination of their abilities to multi-drug resistance. Serratia fonticola and Pantoea sp. isolated from urine catheterized patients who were hospitalized in Iraqi hospitals. They were then examined for detection of biofilm formation phenotypically by congo red and tissue culture plate methods and genetically by detecting SmaI and EsaI genes (quarm sensing genes) in Serratia fonticola and Pantoea sp. respectively by using polymerase chain reaction method and tested for antimicrobial susceptibility by disc diffusion and VITEK2 system according to Clinical and Laboratory Standards Institute (CLSI). Serratia fonticola at 3 and Pantoea sp. at 4 isolates revealed to possess the ability of forming biofilm which contained SmaI and EsaI genes with $100 \%$ resistance to most tested antibiotics except imipenem and azithromycin. SmaI and EsaI genes are present in Serratia fonticola and Pantoea sp. respectively, and are responsible for biofilm formation and considered as indicator; biofilm formation is a strong cause of multidrug resistance in bacteria.
\end{abstract}

Keywords: Biofilm; Serratia fonticola; Pantoea sp.; Urinary catheter; Multidrug resistance

\section{Introduction}

Biofilm is an aggregate of microorganisms such as bacteria and the attachment to biotic surface which is protected by an extracellular polymer matrix composed of polysaccharides and extracellular DNA; it has widespread implications in the medical field [1]. According to the Center for Disease Control (CDC), the rate of bacterial infection associate with biofilm formation was estimated to be $65 \%$ of all infections, while the National Institutes of Health (NIH) estimated $80 \%$. Many microorganisms form biofilms, including fungi and bacteria. The most common bacterial species as causative organisms of biofilm in urinary catheter are Enterobacteriaceae, such as (Serratia and Pantoea sp.). Recently some bacterial strains have entered the 
world of biofilm, including Pantoea sp.; the first report concerning this bacterium was published in 1972, and it was previously named Enterobacter and Erwinia, belonging to the Enterobacteriaceae family [2]. It is an opportunistic pathogen that can cause human diseases by two ways; wound contaminated with plant materials or nosocomial infections [3]. Hospitalized patients, especially those with immunocompromised infections, may be exposed to contaminated equipment or fluids with these bacteria to cause infection $[4,5]$.

Serratia sp. is considered an opportunistic human pathogen, as having achieved a great number of cases of nosocomial infections with the serious problem of multi-drug resistance (MDR) [6]. A large number of Serratia spp. are found to be isolated from urinary tract clinical samples [7, 8]. Manikandan et al. reported that Serratia sp. were the least dominant uropathogen causing urinary tract infection (UTI) [9].

\section{Experimental \\ Materials \\ Collection of specimens}

Twenty-eight biofilm bacterial isolates were isolated from urine samples collected from catheterized patients from four Iraqi hospitals (Al Diwaniya Educational, Al Hilla Eductitional, Al Qassim and Al Hashimiya Hospitals) during the period from February to April 2017 in sterile tubes of $10 \mathrm{~mL}$ and then transferred to laboratory immediately.

\section{Bacterial isolate}

S. fonticola and Pantoea sp. were isolated from urine of catheterized patients which were identified and diagnosed by the automated system VITEK 2 (bioMerieux, Marcy I'Etoile, France) to achieve final diagnostics and identification of the species level.

\section{Reference bacterial strain:}

Serratia marcescens and E. coli were diagnosed by biochemical tests as reference strains to Serratia fonticola and Pantoea sp., respectively.

\section{Biofilm diagnosis}

To detect the biofilm forming bacteria, three methods were employed as follows.

\section{Congo red agar method (quality method)}

According to Freeman, et al. [10], black crystalline colonies were considered as strong biofilm producers, dark colonies without dry crystalline colonies as moderate biofilm producers and dark pink colonies as non-biofilm producers.

\section{Tissue culture plate method}

The test was carried out according to Christensen, et al. [11]. The mean of the absorbance value from replicate wells was read; the biofilm degree was calculated according to the following equation:

Biofilm degree $=$ Mean $\mathrm{OD}_{630}$ of tested bacteria -

Mean $\mathrm{OD}_{630}$ of control,

where $\mathrm{OD}_{630}$ means optical density at $630 \mathrm{~nm}$.

The results were interpreted in Table 1.

Table 1 Classification based on OD values

\begin{tabular}{ccc}
\hline Mean OD value & Adherence & Biofilm formation \\
\hline$<0.120$ & Non & Non/weak \\
$0.120 \sim 0.240$ & Moderate & Moderate \\
$>0.240$ & Strong & Strong \\
\hline
\end{tabular}

Note: $\mathrm{OD}=$ optical density

\section{Genetic diagnosis by polymerase chain reaction (PCR) method}

PCR technique was performed for biofilm formation genes, i.e. smaI and EsaI genes in Serratia sp. and Pantoea sp., respectively. The primers were designed by using National Center for Biotechnology Information (NCBI) gene sequence data base and primer 3 plus design. This primer was provided by Bioneer, South Korea (Table 2).

\section{DNA extraction}

DNA extraction was carried out according to manufactured instructions of commercial DNA extraction kit (Presto Mini-DNA Bacteria Kit. Geneaid Biotech Ltd. USA). Then, extracted DNA was estimated by the nanodrop device at $260 / 280 \mathrm{~nm}$.

\section{PCR master mix preparation}

PCR master mix was prepared from Accu-Power ${ }^{\circledR}$ PCR-PreMix-Kit master mix reagent according to the company directions (Table 3).

The PCR mixture revealed in Table 3 was placed in AccuPower PCR-PreMix that contained all PCR components (Taq DNA polymerase, dNTPs, and 10 PCR buffers). Then, all the PCR tubes were transferred into vortex vibration for $3 \mathrm{~min}$ and transferred into thermocycler apparatus (MyGene, Bioneer, Korea). 
Table 2 Polymerase chain reaction (PCR) primers and their sequence with GenBank codes

\begin{tabular}{cccc}
\hline Primer & & Sequence 5'-3' & Size of PCR amplicon (bp) \\
\hline \multirow{2}{*}{ EsaI gene } & $\mathrm{F}$ & TTTTGCCACCGCGTCAAAAC & 127 \\
& $\mathrm{R}$ & TGGCGTATCGTTGCTGAATC & Pantoea sp. \\
Smal gene & $\mathrm{F}$ & TCACGTCATTTGCAGCTTGC & 73 \\
& $\mathrm{R}$ & ATTGTTGAACACGCCATCGC & Serratia sp. \\
\hline
\end{tabular}

Table 3 Company instructions for polymerase chain reaction (PCR) master mix

\begin{tabular}{cc}
\hline PCR master mix & Volume $(\mu \mathrm{L})$ \\
\hline DNA template & 5 \\
Forward primer $(10$ pmol $)$ & 1.5 \\
Reverse primer $(10$ pmol $)$ & 1.5 \\
PCR water & 12 \\
Total volume & 20 \\
\hline
\end{tabular}

\section{PCR thermocycler conditions}

Conditions of the PCR thermocycler are listed in Table 4.

\section{PCR product analysis}

The products of PCR were analyzed by electrophoresis in a $1 \%$ agarose gel then stained with ethidium bromide and observed under a ultra-violet (UV) transilluminator.

\section{Antimicrobial susceptibility testing}

The test for antimicrobial susceptibility was done by the following two methods.

\section{Disc diffusion method}

According to Clinical and Laboratory Science Institute (CLSI), 2015 [12], the test was carried out on Müller-Hinton agar with antibiotics disc listed in Table 5.

\section{Minimum inhibitory concentration (MIC)}

The testing was done by using VITEK 2 AST system for antibiotics including ampicillin/clavulanic, cefazolin, ceftazidime, ceftriaxone, cefepime, ertapenem, imipenem, gentamicin, tobramycin, ciprofloxacin, levofloxacin, nitrofurantoin, trimethoprim/sulfamethoxazole.

The results in both methods were regarded as sensitive, intermediate or resistant based on the Clinical and Laboratory Science Institute (CLSI) standard guidelines, M100, 2017 [13].

\section{Statistical analysis}

All experiments were carried out in triplicate to validate the reproducibility of experiments. Statistical analysis was done by using one way ANOVA at p-value 0.05 by SPSS Statistics 24.0 software.

\section{Results and Discussion}

The results revealed that Pantoea sp. were the second predominant biofilm forming bacterium at 4 isolates (14\%) followed by $S$. fonticola at 3 isolates (10.7\%) (Fig. 1) which formed biofilm from 65 urine sample. Pantoea sp. belong to the Enterobacteriaceae; it was previously named Enterobacter and Erwinia [14]. It is an opportunistic pathogen that can cause diseases to hospitalized patients, especially those with immune compromised infections when exposed to contaminated equipment or fluids with these bacteria [3]. Recently, Pantoea sp. isolated from humans were considered as an opportunistic pathogen associated with contaminated catheters $[15,16]$. Some species drive different gene expressions by quorum sensing

Table 4 Conditions of (polymerase chain reaction) PCR thermocycler

\begin{tabular}{cccc}
\hline PCR step & Temperature $\left({ }^{\circ} \mathrm{C}\right)$ & Time $(\mathrm{min})$ & Repeat cycle \\
\hline Initial denaturation & 95 & 3 & 0.5 \\
Denaturation & 95 & 0.5 & 30 \\
Annealing & 58 & 0.5 & 5 \\
Extension & 72 & 5 & Hold \\
Final extension & 72 & 4 & 1 \\
Melting & 4 &
\end{tabular}


Table 5 Antibiotics disc with potency and the manufacturer company

\begin{tabular}{cccc}
\hline Antibiotics & Symbol & Potency & Manufacturer \\
\hline Amikacin & AK & $30 \mu \mathrm{g}$ & \\
Azithromycin & AZM & $15 \mu \mathrm{g}$ & \\
Carbenicillin & PY & $100 \mu \mathrm{g}$ & \\
Clindamycin & DA & $2 \mu \mathrm{g}$ & Bioanalysis, \\
Imipenem & IPM & $10 \mu \mathrm{g}$ & \\
Penicillin G & P & $10 \mathrm{IU}$ & \\
Tobramycin & TOB & $10 \mu \mathrm{g}$ & \\
\hline
\end{tabular}

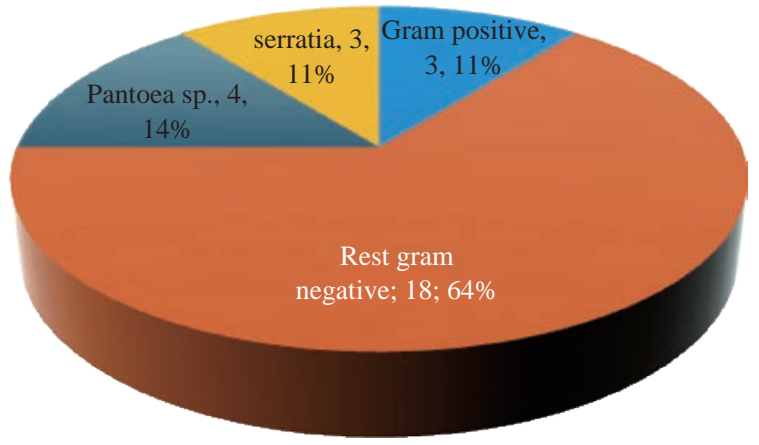

Fig. 1 Biofilm forming bacterial isolates.

ability which hence controls physiological activities [17] involving biofilm formation. Many studies managed to isolate Pantoea sp. from UTI patients at the rate of $10 \%$ to $21.4 \%[18,19]$. Meanwhile, a large number of Serratia sp. were isolated from urinary tract clinical samples [7, 8]. Manikandan and coworkers [9] reported that Serratia sp. were the least dominant uropathogen causing UTI, although the ability to form biofilm may contribute to its pathogenicity to make them clinically important [20]. It has flagella-mediated swimming and swarming motility which are associated with biofilm formation and facilitate the contact between bacterial cells and solid materials [21]. That may explain the ability of Serratia sp. to form biofilm phenomenon on urinary catheter. Biofilm production has been reported for several Serratia sp. [22].

The results revealed most bacterial isolates that showed biofilm formation belonged to the Enterobacteriaceae family. Biofilm formation associated with urinary catheter infections are mostly caused by Enterobacteriaceae microflora, gram negative rods (Escherichia coli, Klebsiella pneumonia, Enterobacter sp., Pseudomonas aeruginosa, Serratia sp., Proteus mirabilis, etc.) [23], who are thought to be derived largely from the patient's own gut microbiota. The most common routes of urinary tract infection mainly involve fecal flora ascending to the bladder and kidneys via the urethra. The bacteria that colonize the distal urethra attach to the external surface or lumen of the catheter, as it is inserted into the bladder [24, 25].

The main bacterial virulence factors involve in biofilm formation are urease production and different types of motility. Most Enterobacteriaceae members are urease positive, the most significantly, Proteus sp., Klebsiella pneumonia, Pseudomonas aeruginosa and Serratia marcescens [26]. Also, the type of motility can facilitate the movement of bacteria and attach to catheter surface to form biofilm.

\section{Biofilm detection}

Pantoea sp. and S. fonticola isolates that produced biofilm appeared on Congo red media (Fig. 2). The black color was a result of polysaccharide matrix formation during biofilm forming process which was stained by congo red to black color [27]

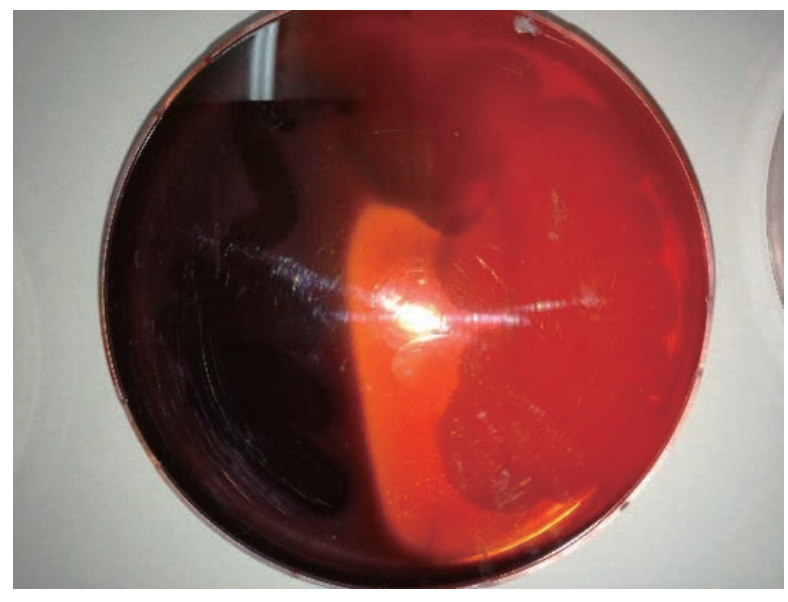

Fig. 2 Congo red agar indicating the biofilm of Pantoea sp. and S. fonticola.

In tissue culture plate (TCP) method, the bacterial isolates showed a strong, moderate and weak or nonbiofilm production. Pantoea sp. and S. fonticola isolates showed strong and moderate biofilm degree, respectively, with significant difference at $p$ value $=$ 0.005 (Table 6), after data calculation of OD values obtained for individual biofilm bacterial isolates.

\section{Molecular assay}

The molecular assay was done for Pantoea sp. and Serratia fonticola which were isolated at the first time locally as biofilm forming bacteria from urinary catheterized patients.

\section{Pantoea sp.}

PCR analysis of Pantoea sp. revealed that the four Pantoea isolates had EsaI gene (Fig. 3) with the 
Table 6 Statistical analysis of optical density (OD) of Pantoea sp. and S. fonticola in tissue culture plate (TCP)

\begin{tabular}{cc}
\hline Biofilm bacteria & OD mean \pm SE \\
\hline Pantoea sp. & $0.6597 \pm 0.1462$ \\
S. fonticola & $\mathrm{A}$ \\
& $0.2520 \pm 0.0450$ \\
\hline
\end{tabular}

Note: $\mathrm{SE}=$ stander error; Different capital letters (A, B) mean significant differences $(p \leq 0.05)$ between different optical densities.

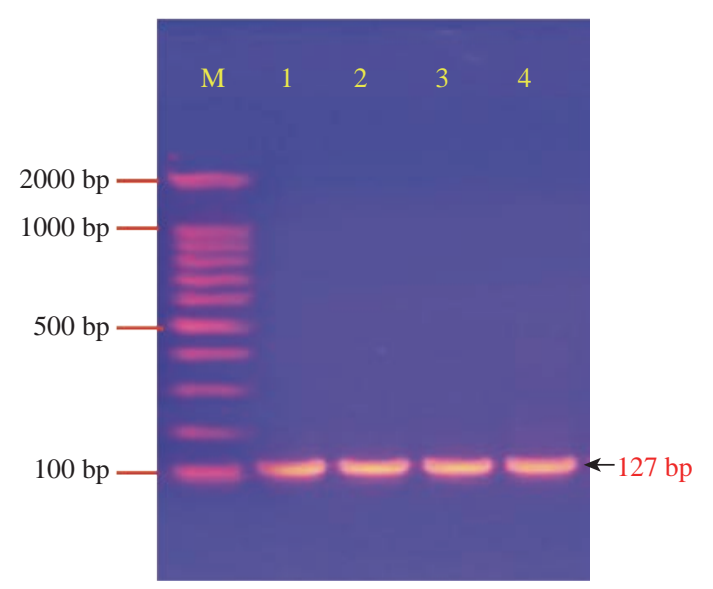

Fig. 3 Agarose gel electrophoresis of PCR assay showing some positive results of biofilm formation by EsaI gene in Pantoea sp. positive isolates: Lane (M) DNA marker (2000 100 bp); Lane (1-4) Positive EsaI at 127 bp PCR product size.

Amplicon size of $127 \mathrm{bp}$, meaning they had the ability to synthesize quorum sensing (QS) signaling molecules $\mathrm{N}$-acylhomoserine lactones (AHLs)) and biofilm formation $[28,29]$. It was pointed out that Pantoea members had been used to synthesize QS which regulated many phenotypes, such as the production of virulence factor, the aggregation of cells and biofilm formation [30]

Pantoea sp. showed QS activity by increasing cell density-dependent exopolysaccharide substance (EPS) synthesis [17, 31]. Exopolysaccharide (EPS) was a major component of biofilm bacterial matrix and a powerful virulence factor which protected the bacterial cell from antibiotics action and hosted immune defense [32]. EsaI quorum sensing gene governed the synthesis of EPS in Pantoea sp. to appropriate bacterial adhesion and biofilm formation [33]. EsaI gene was considered a typical N-acyl-L homoserine lactone (AHL) synthase which catalyzed synthesis of N-3-oxo-hexanoyl homoserine lactone [34].

Other studies suggested that the occurrence of EsaI gene mutation led to the lack of adhesion ability and biofilm formation by inhibiting AHL synthesis [33]. Also, the degradation of QS by chemical or biological inhibitors might inhibit biofilm formation [35].
Most of the studies about Pantoea sp. EsaI gene as cited were those associated with plant diseases only; there was no study about Pantoea EsaI gene in human diseases.

\section{Serratia fonticola:}

Fig. 4 shows the amplification of the SmaI gene (quorum sensing gene) in S. fonticola isolates. The results revealed that bacterial isolates were positive to SmaI gene, with the Amplicon size as of $73 \mathrm{bp}$.

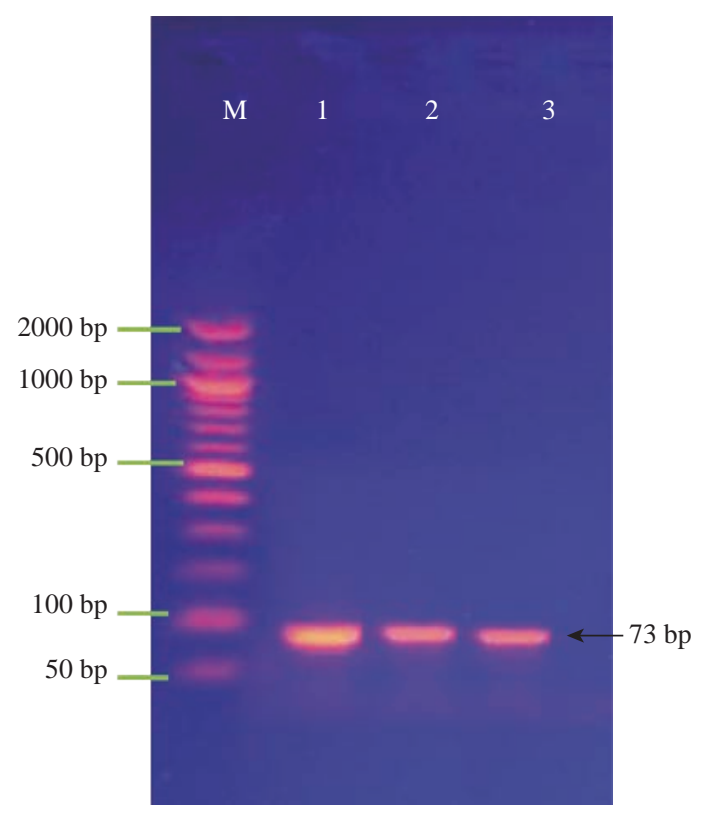

Fig. 4 Agarose gel electrophoresis of PCR assay showing some positive results of biofilm formation by SmaI gene in Serratia sp. positive isolates: Lane (M) DNA marker (2000 50 bp); Lane (1-3) Positive SmaI at 73 bp PCR product size.

Biofilm formation and swarming motility of Serratia sp. were regulated by quorum sensing [36]. SmaI was a quorum sensing-regulated gene that was involved in biofilm development [37], which regulated the activity of hemolysis, swarming motility and biofilm formation [38, 39]. QS Signaling molecules are also called autoinducers; they are small chemical molecules having a major role in attaching and inducing bacterial cells to aggregate with each other to form biofilm [40, 41].

Coulthurst et al. showed that biofilm formation in Serratia sp. was dependent on SmaI gene, detected by TCP as a biofilm formation indicator [38]. The Biofilm form was controlled by AHL-dependent quorum sensing in this bacteria [37]. In 2014, it was provided evidence for the first time that quorum sensing activity was present in Serratia fonticola by confirming a three short chains of AHLs [8]. 
Serratia sp. expressed QS gene (SmaI) by utilizing C4- homoserine lactone (C4-HSL) as signal molecules and directing the production of various virulence factors like prodigiosin, protease, hemolysin production, and most significantly, biofilm formation [42]. Quorum sensing system (SmaI) appeared to play a role in regulating biofilm production for Serratia sp., as described above.

\section{Antimicrobial susceptibility testing}

The current study focused on determining and evaluating antibiotic resistance in biofilm forming urobacteria (Serratia fonticola and Pantoea sp.) by two methods: disc diffusion and MIC in VITEK2.

\section{Disc diffusion and VITEK AST method}

These methods were applied to test the biofilm forming bacteria Staphylococcus lentus, Serratia fonticola and Pantoea sp.

S. fonticola and Pantoea sp. were revealed as multidrug resistant. Antimicrobial susceptibility testing was carried out by two methods: disc diffusion and MIC by VITEK AST. In disc diffusion method, all isolates of three Serratia and four Pantoea sp. (Fig. 5, 7 and Fig. $6,8)$ respectively displayed resistance rate of $100 \%$
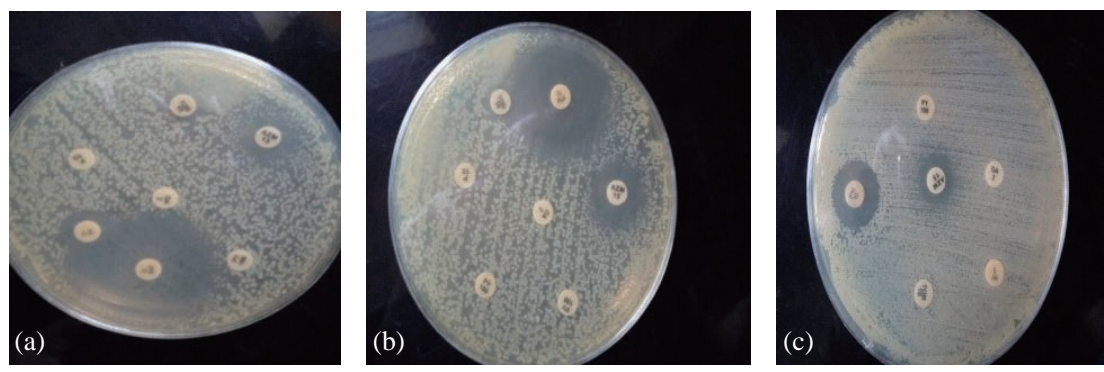

Fig. 5 Testing of antibiotic resistance to $S$. fonticola isolates by disc diffusion method. (a) S. fonticola 1; (b) S. fonticola; (c) S. fonticola 3 .
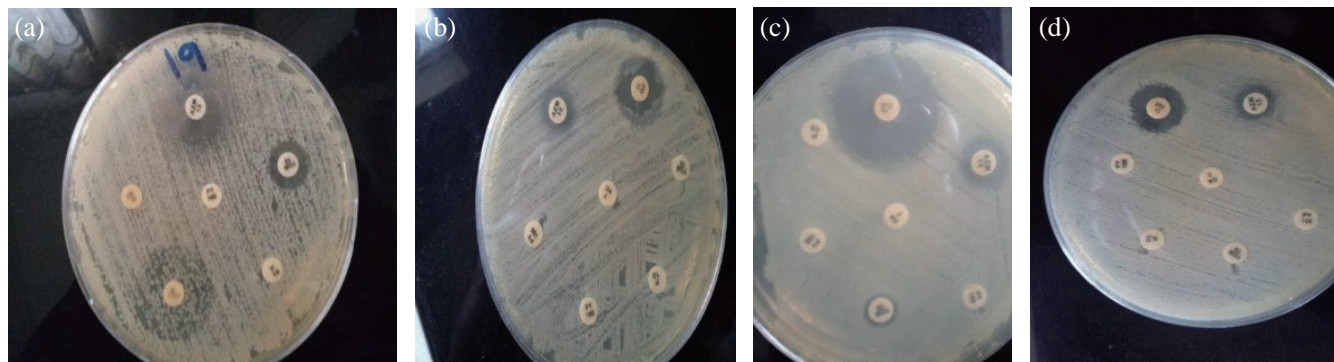

Fig. 6 Testing of antibiotic resistance to Pantoea sp. isolates by disc diffusion method. (a) Pantoea 1; (b) Pantoea 2; (c) Pantoea 3; (d) Pantoea 4.

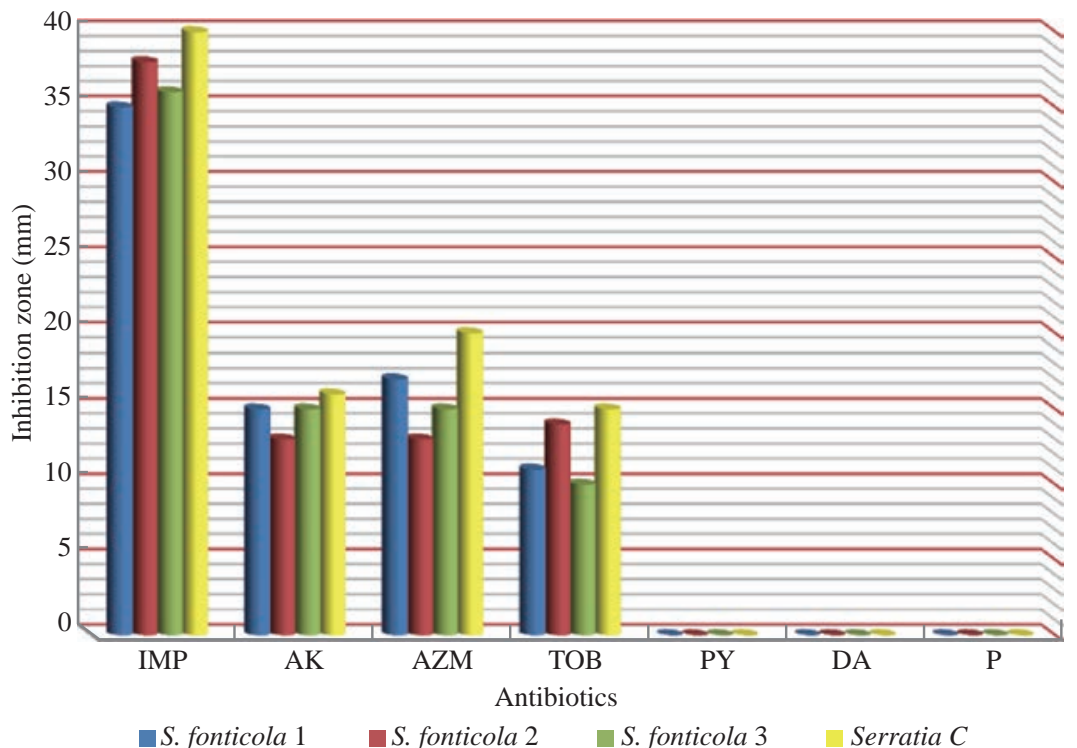

Fig. 7 Zone of inhibition $(\mathrm{mm})$ of different antibiotics in disc diffusion method against $S$. fonticola and S. marcescens as control (IMP = imipenem; $\mathrm{AK}=$ amikacin; $\mathrm{AZM}=$ azithromycin; $\mathrm{TOB}=$ tobramycin; $\mathrm{PY}=$ carbenicillin; $\mathrm{DA}=$ clindamycin; $\mathrm{P}=$ penicillin). 


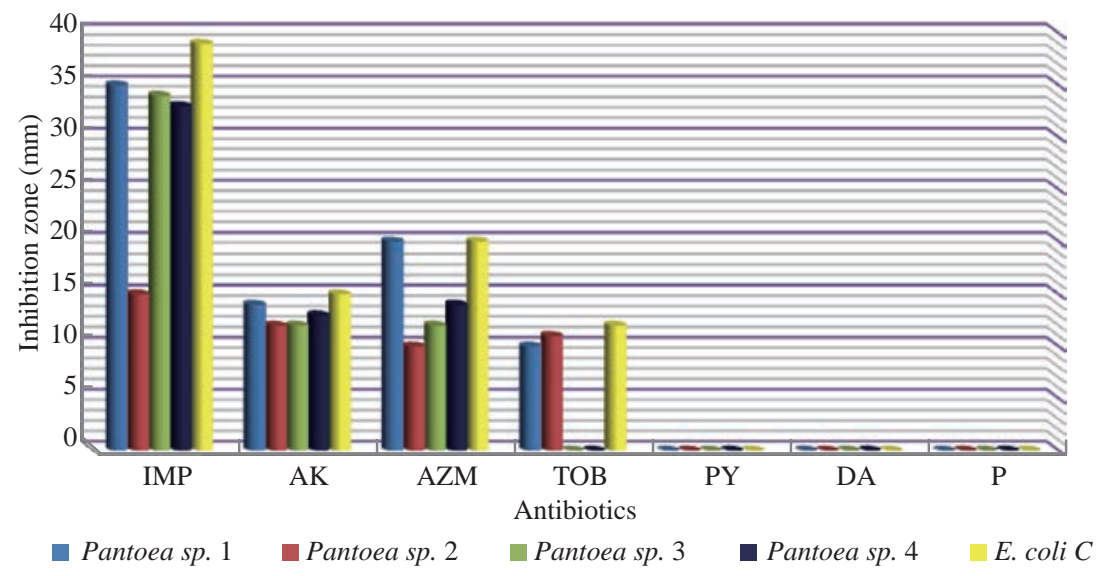

Fig. 8 Zone of inhibition $(\mathrm{mm})$ of different antibiotics in disc diffusion method against pantoea sp. and E. coli as control (IMP = imipenem; $\mathrm{AK}=$ amikacin; $\mathrm{AZM}=$ azithromycin; $\mathrm{TOB}=$ tobramycin; $\mathrm{PY}=$ carbenicillin; $\mathrm{DA}=$ clindamycin; $\mathrm{P}=$ penicillin).

to amikacin, tobramycin, carbenicillin, clindamycin and penicillin. S. fonticola revealed sensitivity rate at $100 \%$ to imipenem and azithromycin, while Pantoea sp. revealed sensitivity rate of $100 \%$ to imipenem and $50 \%$ to azithromycin. On the other hand, the control nonbiofilm bacteria (S. marcescens and E. coli) showed high sensitivity to imipenem, amikacin, azithromycin and tobramycin at the sensitivity rate of $100 \%$ to each one in comparison to S. fonticola and Pantoea sp. sensitivity results respectively.

Statistical analysis of susceptibility to imipenem revealed there was significant difference between Pantoea sp. isolates as well as between the isolates and control at $p<0.05$ (Table 7). Meanwhile, S. fonticola showed a significant difference between its isolates and bacterial control (Table 8) at $p<0.05$.

Some studies pointed out that non-biofilm Pantoea sp. were uniformly susceptible to most antibiotics tested in their studies [14, 43], but biofilm Pantoea sp. were less susceptible to antibiotics than free planktonic bacteria [44].

Table 7 Statistical analysis of susceptibility of biofilm forming of Pantoea sp. isolates and control to imipenem according to inhibition zone

\begin{tabular}{cc}
\hline Bacteria & Inhibition zone + SE \\
\hline Pantoea sp. 1 & $35 \pm 0.577$ \\
& A \\
Pantoea sp. 2 & $15 \pm 0.577$ \\
& B \\
Pantoea sp. 3 & $34 \pm 0.577$ \\
& A \\
Pantoea sp. 4 & $33 \pm 0.577$ \\
Control: E. coli & C \\
\end{tabular}

Note: Values represent mean \pm standard deviation (SE); Different capital letters (A-D) mean significant differences $(p \leq 0.05)$ between different inhibition zones.
Table 8 Statistical analysis of susceptibility of biofilm forming S. fonticola isolates and control to imipenem according to inhibition zone

\begin{tabular}{cc}
\hline Bacteria & Inhibition zone \pm SE \\
\hline S. fonticola1 & $38 \pm 0.577$ \\
S. fonticola2 & $\mathrm{A}$ \\
& $36 \pm 1.15$ \\
S. fonticola3 & $\mathrm{A}$ \\
& $35 \pm 1.15$ \\
S. mercenes & $\mathrm{A}$ \\
\end{tabular}

Note: Values represent mean \pm standard deviation (SE); Different capital letters (A, B) refer to significant differences $(p \leq 0.05)$ between different inhibition zones.

Imipenem was the most effective antibiotic against biofilm forming gram negative bacteria at $100 \%$ sensitivity. It was used to treat complicated urinary tract infections according to CLSI, 2017 [13]. Imipenem was a $\beta$-lactam antibiotic, resistant to $\beta$-lactamase producer bacteria which remained very stable against penicillinase and cephalosporinase bacteria; it had broad spectrum action on gram positive and gram negative bacteria according to CLSI, 2012 [45]. It acted as cell wall synthesis inhibitor by binding to penicillin binding proteins (PBPs) to cause loss of cell wall integrity and cell lysis, leading to rapid bacterial cell death [46].

In VITEK AST analysis, S. fonticola and Pantoea sp. revealed resistance rate at $100 \%$ to most antibiotics in the test as shown in Table 9.

In addition to the reasons above, many factors lie beneath the ability of bacteria to resist antibiotics, one of which is the biofilm. In this study, the biofilm forming bacteria displayed significantly high antibiotic resistance, which was correspondent to other studies [47, 48]. In the biofilm state, it was more difficult for the antibiotics 
Table 9 VITEK2 antibiotic sensitivity test (AST) system results

\begin{tabular}{|c|c|c|c|c|}
\hline \multirow{2}{*}{ Antibiotics } & \multicolumn{2}{|c|}{ S. fonticola } & \multicolumn{2}{|c|}{ Pantoea sp. } \\
\hline & $\mathrm{MIC}(\mu \mathrm{g} / \mathrm{mL})$ & Interp. & $\mathrm{MIC}$ & Interp. \\
\hline Ampicillin/clavulanic & $\geq 32$ & $\mathrm{R}$ & $\geq 32$ & $\mathrm{R}$ \\
\hline Cefazolin & $\geq 64$ & $\mathrm{R}$ & $\geq 64$ & $\mathrm{R}$ \\
\hline Ceftazidime & $\geq 64$ & $\mathrm{R}$ & $\geq 64$ & $\mathrm{R}$ \\
\hline Ceftriaxone & $\geq 64$ & $\mathrm{R}$ & $\geq 64$ & $\mathrm{R}$ \\
\hline Cefepime & $\geq 64$ & $\mathrm{R}$ & 16 & $\mathrm{R}$ \\
\hline Ertapenem & $\geq 0.5$ & $\mathrm{~S}$ & $\geq 8$ & $\mathrm{R}$ \\
\hline Imipenem & $\geq 2$ & $\mathrm{I}$ & $\geq 16$ & $\mathrm{R}$ \\
\hline Gentamicin & $\geq 16$ & $\mathrm{R}$ & $\geq 16$ & $\mathrm{R}$ \\
\hline Tobramycin & $\geq 16$ & $\mathrm{R}$ & $\geq 16$ & $\mathrm{R}$ \\
\hline Ciprofloxacin & $\geq 4$ & $\mathrm{R}$ & 2 & I \\
\hline Levofloxacin & $\geq 8$ & $\mathrm{R}$ & 4 & I \\
\hline Nitrofurantoin & 128 & $\mathrm{R}$ & 128 & $\mathrm{R}$ \\
\hline Trimethoprim/sulfamithaxzol & $\geq 320$ & $\mathrm{R}$ & $\geq 320$ & $\mathrm{R}$ \\
\hline
\end{tabular}

Note: $\mathrm{R}=$ resistant; $\mathrm{I}=$ intermediate; $\mathrm{MIC}=$ minimum inhibitory concentration; Interp. = interpreter.

to diffuse into the bacteria, and the compounds of the matrix bound to the antibiotics also increased the difficulty [49]. The extracellular DNA displayed antibiotic chelating activity [50]. In addition to different metabolic states and as a result of grades of nutrients, oxygen viability depending on their depth inside the biofilm layers also affected bacterial susceptibility to antibiotics [47]. Gene transfer played a major role in antibiotic resistance by sharing genetic information via horizontal gene transfer among bacterial cells forming biofilm including antibiotic resistant ability [51].

\section{Conclusions}

In the present study, it was found that the EsaI and SmaI genes were present in Pantoea sp. and $S$. fonticola respectively, and were responsible for biofilm formation in these bacteria as biofilm regulatory genes. These genes could be considered as the indicator of biofilm formation. And the biofilm formation was a strong cause of multi-drug resistance in bacteria.

\section{Conflict of Interests}

The authors declare that no competing interest exists.

\section{References}

[1] S. Neethirajan, M.A. Clond, and A. Vogt, Medical biofilms - nanotechnology approaches. Journal of biomedical nanotechnology, 2014, 10(10): 2806-2827.

[2] E. Bottone, S.S. Schneierson, Erwinia species: an emerging human pathogen. American Journal of Clinical Pathology, 1972, 57(3): 400-405.

[3] J. Dutkiewicz, B. Mackiewicz, M.K. Lemieszek, et al., Pantoea agglomerans: a mysterious bacterium of evil and good. Part III. Deleterious effects: infections of humans, animals and plants. Annals of Agricultural and Environmental Medicine, 2016, 23(2).

[4] A. Shubov, P. Jagannathan, and P. Chin-Hong, Pantoea agglomerans pneumonia in a heart-lung transplant recipient: case report and a review of an emerging pathogen in immunocompromised hosts. Transplant Infectious Disease, 2011, 13(5): 536-539.

[5] I. Boszczowski, J.N. De Almeida Júnior, E.P. De Miranda, et al., Nosocomial outbreak of Pantoea agglomerans bacteraemia associated with contaminated anticoagulant citrate dextrose solution: new name, old bug? Journal of Hospital Infection, 2012, 80(3): 255-258.

[6] I.R.A. Mun, A.H. Alsakini, and S.M. Karim, Correlation between prodigiocin, biofilm formation and drug resistant in virulent Serratia marcescens. Journal of Al-Nahrain University-Science, 2013, 16(4): 188-197.

[7] I. Henriques, R.T.J. Ramos, R.A. Baraúna, et al., Draft genome sequence of Serratia fonticola UTAD54, a carbapenem-resistant strain isolated from drinking water. Genome announcements, 2013, 1(6): e00970-13.

[8] R. Ee, Y.L. Lim, K.K. Tee, et al., Quorum sensing activity of Serratia fonticola strain RB-25 isolated from an exlandfill site. Sensors, 2014, 14(3): 5136-5146.

[9] S. Manikandan, S. Ganesapandian, S. Singh, et al., 
Antimicrobial susceptibility pattern of urinary tract infection causing human pathogenic bacteria. Asian Journal of Medical Sciences, 2011, 3(2): 56-60.

[10] D. Freeman, F. Falkiner, and C. Keane, New method for detecting slime production by coagulase negative staphylococci. Journal of Clinical Pathology, 1989, 42(8): 872-874.

[11] G.D. Christensen, W. Simpson, J. Younger, et al., Adherence of coagulase-negative staphylococci to plastic tissue culture plates: A quantitative model for the adherence of staphylococci to medical devices. Journal of clinical microbiology, 1985, 22(6): 996-1006.

[12] CLSI, Performance Standards for Antimicrobial Disk Susceptibility Tests; Approved Standard M02/ A12, $12^{\text {th }}$ Ed. Clinical and Laboratory Standards Institute, 2015.

[13] CLSI, Performance Standards for Antimicrobial Susceptibility Testing M100. Clinical and Laboratory Standards Institute, 2017.

[14] R. Kazancioglu, B. Buyukaydin, M. Iraz, et al., An unusual cause of peritonitis in peritoneal dialysis patients: Pantoea agglomerans. The Journal of Infection in Developing Countries, 2014, 8(07): 919-922.

[15] A. Delétoile, D. Decre, S. Courant, et al., Phylogeny and identification of Pantoea species and typing of Pantoea agglomerans strains by multilocus gene sequencing. Journal of clinical microbiology, 2009, 47(2): 300-310.

[16] L.S. AbdAlhussen, M.F. Darweesh, Prevelance and antibiotic susceptibility patterns of Pantoea spp. isolated form clinical and environmental sources in Iraq. International Journal of ChemTech Research, 2016, 9(8): 430-437.

[17] W.-S. Tan, M. Yunos, N. Yusrina, et al., Pantoea sp. isolated from tropical fresh water exhibiting N-acyl homoserine lactone production. The Scientific World Journal, 2014, 2014.

[18] A.T. Cruz, A.C. Cazacu, and C.H. Allen, Pantoea agglomerans, a plant pathogen causing human disease. Journal of Clinical Microbiology, 2007, 45(6): 19891992.

[19] A. Büyükcam, Ö. Tuncer, D. Gur, et al., Clinical and microbiological characteristics of Pantoea agglomerans infection in children. Journal of Infection and Public Health, 2017.

[20] T. Hirata, E. Kitahara, and Y. Nakagawa, Novel screening of antibiotic-sensitive biofilm-forming mutants by utilizing a product of Serratia marcescens. Microbes and Environments, 2006, 21(3): 148-153

[21] L. Eberl, S. Molin, and M. Givskov, Surface motility of Serratia liquefaciens MG1. Journal of Bacteriology, 1999, 181(6): 1703-1712.

[22] X. Liu, J. Jia, R. Porat, et al., Characterisation of two quorum sensing systems in the endophytic Serratia plymuthica strain G3: Differential control of motility and biofilm formation according to life-style. BMC microbiology, 2011, 11(1): 26.

[23] P. Tenke, B. Kovacs, M. Jäckel, et al., The role of biofilm infection in urology. World Journal of Urology, 2006. 24(1): 13

[24] T.M. Hooton, Pathogenesis of urinary tract infections: an update. Journal of Antimicrobial Chemotherapy, 2000, 46(suppl_1): 1-7.

[25] S.Á Jacobsen, D. Stickler, H. Mobley, et al., Complicated catheter-associated urinary tract infections due to Escherichia coli and Proteus mirabilis. Clinical Microbiology Reviews, 2008, 21(1): 26-59.

[26] V. Hola, F. Ruzicka, The formation of poly-microbial biofilms on urinary catheters. Urinary tract infections. InTech, 2011.

[27] S. Bose, M. Khodke, S. Basak, et al., Detection of biofilm producing staphylococci: Need of the hour. Journal of
Clinical and Diagnostic Research, 2009, 3(6): 1915-1920.

[28] T. Morohoshi, Y. Ogata, and T. Ikeda, Cell aggregation is negatively regulated by $\mathrm{N}$-acylhomoserine lactonemediated quorum sensing in Pantoea ananatis SK-1. Journal Of Bioscience and Bioengineering, 2011, 112(6): 566-569.

[29] S.B. Von Bodman, D.R. Majerczak, and D.L. Coplin, A negative regulator mediates quorum-sensing control of exopolysaccharide production in Pantoea stewartii subsp. stewartii. Proceedings of the National Academy of Sciences, 1998. 95(13): 7687-7692.

[30] T. Morohoshi, Y. Nakamura, G. Yamazaki, et al., The plant pathogen Pantoea ananatis produces $\mathrm{N}$-acylhomoserine lactone and causes center rot disease of onion by quorum sensing. Journal of Bacteriology, 2007, 189(22): 83338338.

[31] N.Y.M. Yunos, W.-S. Tan, N. I. Mohamad, et al., Discovery of Pantoea rodasii strain ND03 that produces N-(3-oxo-hexanoyl)-L-homoserine lactone. Sensors, 2014, 14(5): 9145-9152.

[32] T.D. Minogue, A.L. Carlier, M.D. Koutsoudis, et al., The cell density - dependent expression of stewartan exopolysaccharide in Pantoea stewartii ssp. stewartii is a function of EsaR - mediated repression of the rcsA gene. Molecular microbiology, 2005. 56(1): 189-203.

[33] M.D. Koutsoudis, D. Tsaltas, T.D. Minogue, et al., Quorum-sensing regulation governs bacterial adhesion, biofilm development, and host colonization in Pantoea stewartii subspecies stewartii. Proceedings of the National Academy of Sciences, 2006, 103(15): 5983-5988.

[34] W.T. Watson, T.D. Minogue, D.L. Val, et al., Structural basis and specificity of acyl-homoserine lactone signal production in bacterial quorum sensing. Molecular Cell, 2002, 9(3): 685-694.

[35] T.B. Rasmussen, T.T. Bjarnshol, M.E. Skindersoe, et al., Screening for quorum-sensing inhibitors (QSI) by use of a novel genetic system, the QSI selector. Journal of Bacteriology, 2005. 187(5): 1799-1814.

[36] S. Horinouchi, Cell-to-cell communications among microorganisms. Comprehensive Natural Products II: Chemistry and Biology, 2010, 4: 283-337.

[37] R. Van Houdt, M. Givskov, and C.W. Michiels, Quorum sensing in Serratia. FEMS Microbiology Reviews, 2007, 31(4): 407-424.

[38] S.J. Coulthurst, N.R. Williamson, A.K. Harris, et al., Metabolic and regulatory engineering of Serratia marcescens: mimicking phage-mediated horizontal acquisition of antibiotic biosynthesis and quorum-sensing capacities. Microbiology, 2006, 152(7): 1899-1911.

[39] S.D. Mahlen, Serratia infections: from military experiments to current practice. Clinical Microbiology Reviews, 2011, 24(4): 755-791.

[40] C. Fuqua, M.R. Parsek, and E.P. Greenberg, Regulation of gene expression by cell-to-cell communication: acylhomoserine lactone quorum sensing. Annual Review of Genetics, 2001, 35(1): 439-468.

[41] M.B. Miller, B.L. Bassler, Quorum sensing in bacteria. Annual Reviews in Microbiology, 2001, 55(1): 165-199.

[42] Rice, S., K. Koh, S. Queck, et al., Biofilm formation and sloughing in Serratia marcescens are controlled by quorum sensing and nutrient cues. Journal of Bacteriology, 2005, 187(10): 3477-3485.

[43] J. Mardaneh, M.M.S. Dallal, Isolation, identification and antimicrobial susceptibility of Pantoea (Enterobacter) agglomerans isolated from consumed powdered infant formula milk (PIF) in NICU ward: First report from Iran. Iranian Journal of Microbiology, 2013, 5(3): 263.

[44] R.M. Donlan, Role of biofilms in antimicrobial resistance. ASAIO Journal, 2000, 46(6): S47-S52.

[45] CLSI, Methods for Dilution Antimicrobial Susceptibility 
Tests for Bacteria that Grow Aerobically; Approved Standard, $9^{\text {th }}$ Ed., CLSI Document M07-A9. Clinical and Laboratory Standards Institute, 2012.

[46] D. Mehta, British National Formulary, Vol. 49. Pharmaceutical Press, 2005.

[47] F. Corona, J.L. Martinez, Phenotypic resistance to antibiotics. Antibiotics, 2013, 2(2): 237-255.

[48] K. Smith, I.S. Hunter, Efficacy of common hospital biocides with biofilms of multi-drug resistant clinical isolates. Journal of Medical Microbiology, 2008. 57(8): 966-973.

[49] A. Corbin, B. Pitts, A. Parker, et al., Antimicrobial penetration and efficacy in an in vitro oral biofilm model. Antimicrobial Agents and Chemotherapy, 2011, 55(7): 3338-3344.
[50] K. Lewis, Persister cells. Annual Review of Microbiology, 2010, 64: 357-372

[51] N. Bagge, M. Hentzer, J.B. Andersen, et al., Dynamics and spatial distribution of $\beta$-lactamase expression in Pseudomonas aeruginosa biofilms. Antimicrobial Agents and Chemotherapy, 2004, 48(4): 1168-1174.

Copyright $($ Shaimaa Obaid Hasson, Adnan Hamad A1Hamadani, and Ibtisam Habeeb Al-Azawi. This is an openaccess article distributed under the terms of the Creative Commons Attribution License, which permits unrestricted use, distribution, and reproduction in any medium, provided the original author and source are credited. 